\title{
Effective field methods with correlations in magnetic systems
}

\author{
R. Dekeyser and G. Kamieniarz ${ }^{1}$ \\ Instituut voor Theoretische Fysica, Katholieke Universiteit Leuven, Celestijnenlaan 200 D, B-3001 Heverlee, Belgium
}

\begin{abstract}
The self-consistency conditions inherent in the mean field approximation are extended to take account of pair correlations. It is shown on the linear $X Y$-model how this leads to improved estimates from finite-size calculations.
\end{abstract}

The molecular field approximation (MFA) [1] is still a valuable tool as well for obtaining a qualitative understanding of phase diagrams as for deriving quantitative estimates of nonsingular thermodynamic properties, whenever exact solutions are impossible. Various correction schemes have been proposed in order to improve the accuracy of such estimates. We will briefly review some of these schemes and show how they may be extended in such way that also the correlations, which are completely neglected in the standard MFA, are taken into account. We will also show how this improved method may be applied to quantum mechanical models of magnetism, where it may be combined with other approaches, like finite size calculations, to obtain accurate estimates of thermodynamical quantities.

In order to present the method in a general framework, we suppose that we want to study a system with short range interactions, which can be divided into a finite cluster $\Omega$, its boundary $\partial \Omega$ and $\bar{\Omega}$, the complement of $\Omega \cup \partial \Omega$, in such a way that the Hamiltonian can be written as

$-\beta \mathscr{H}=H_{0}(\Omega, \partial \Omega)+H_{1}(\partial \Omega, \bar{\Omega})$.

Denoting the degrees of freedom by the variables $\sigma \in \Omega$ and $\tau \in \partial \Omega$, we would like to find an efficient way for estimating the thermal expectation value $\langle A\rangle$ of an operator $A(\sigma, \tau)$.

The first category of approximations either fixes the boundary $\partial \Omega$ in a particular state, or it uses some probability distribution $P(\tau)$ for the boundary states. The approximation then claims that

$\langle A\rangle \approx \sum_{\tau} P(\tau) \frac{\sum_{\sigma} A \mathrm{e}^{H_{0}}}{\sum_{\sigma} \mathrm{e}^{H_{0}}}$,

where the Standard MFA consists in choosing

$P(\tau)=\prod_{i} \delta\left(\tau_{i}-m\right)$

\footnotetext{
${ }^{1}$ Present address: Faculty of Applied Physics, TU Delft, The Netherlands.
}

and $m$ is calculated self-consistently from $m=\langle\sigma\rangle$. A more sophisticated choice for the Ising model would be

$P(\tau)=\prod_{i}\left[\frac{1+m}{2} \delta\left(\tau_{i}-1\right)+\frac{1-m}{2} \delta\left(\tau_{i}+1\right)\right]$.

A second category of approximations [2] starts from the idea that one may correct for the neglect of $\bar{\Omega}$ by introducing extra molecular fields in the Hamiltonian, acting on the boundary $\partial \Omega$ :

$\langle A\rangle \approx \frac{\sum_{\tau} \sum_{\sigma} A \mathrm{e}^{H_{0}+H^{\prime}(\tau)}}{\sum_{\tau} \sum_{\sigma} \mathrm{e}^{H_{0}+H^{\prime}(\tau)}}$.

The standard Bethe approximation supposes

$\mathrm{e}^{H^{\prime}}=\prod_{i}\left(1+a \tau_{i}\right)$

where $a$ is again determined self-consistently from $\langle\sigma\rangle=\langle\tau\rangle$. More sophisticated versions have been used by Suzuki [3] under the name "Super-effective-field theory".

For classical systems, this scheme would be exact if one could use the correct $H^{\prime}$, given by

$\mathrm{e}^{H^{\prime}(\tau)}=\operatorname{Tr}_{\bar{\Omega}} \mathrm{e}^{H_{1}(\partial \Omega, \bar{\Omega})}$.

It is possible to improve on both schemes by including in $P(\tau)$ or in $H^{\prime}(\tau)$ some correlations between the $\tau$-variables. Two strategies can be followed:

(a) Self-consistent conditions can be imposed to determine free parameters in $P$ or $H^{\prime}$, in analogy with the usual mean field approximation. Correlations inside $\Omega$ may, e.g., be imposed to be equal to equivalent correlations inside $\partial \Omega$. Besides nearest-neighbour correlations, also next-nearest neighbours may be considered or higher-order correlations.

(b) Approximate expressions can be used for the correlations induced through $H_{1}$. This can be done by performing standard series expansion techniques on eq. (7).

The method with the effective fields in $H^{\prime}$ of eq. (5) proves to be the most efficient. This is most pronounced in one-dimensional problems, where the method yields exact results for classical systems. This 
success is due to the fact that $H^{\prime}$ contains only those correlations that come from interactions through $\bar{\Omega}$ and not those from interactions through $\Omega$. The correlation between the leftmost and rightmost site of a finite segment of a line, however, is completely due to interactions through this segment. This makes the Bethe scheme exact in one dimension, where $H^{\prime}$ consists only of a simple molecular field $h\left(\sigma_{1}+\sigma_{N}\right)$.

Equation (7) is no longer exact for quantum mechanical models, because the factor $\exp \left(H_{1}\right)$ can in general not be extracted from the Boltzmann factor. Instead, we should define

$\mathrm{e}^{H_{0}+H^{\prime}}=\operatorname{Tr}_{\bar{\Omega}} \mathrm{e}^{H_{0}(\Omega, \partial \Omega)+H_{1}(\partial \Omega, \bar{\Omega})}$.

As a consequence this means that, in contrast to the classical case, $H^{\prime}$ may also depend on the variables $\sigma$ from the interior of the cluster $\Omega$. Nevertheless, one still expects the strongest contributions to $H^{\prime}$ to be found at or around the boundary $\partial \Omega$.

Approximate expressions for $H^{\prime}$ may be derived from eq. (8) using, e.g., the Baker-Campbell-Hausdorff formula or Feynman's identity. We will, however, follow the path which is more in the spirit of the MFA by guessing through symmetry arguments the form of the most important terms in $H^{\prime}$, and by determining its parameters through self-consistency calculations. Since we hope to be able to improve through such approach the numerical extrapolations obtained from finite size calculations for one-dimensional magnetic systems like CHAB [4], we first want to test the efficiency of the method by applying it to a simple model like the quantum $X Y$-chain.

For the simple $X Y$-Hamiltonian without external field, we can argue that for symmetry reasons no external field can appear in $H^{\prime}$. The first contribution that can be expected in $H^{\prime}$ is an extra $X Y$-interaction between the first and the second spin in a finite seg-

\section{Table 1}

Results for the specific heat per site from standard method and from effective field calculations for the $X Y$-model on a chain of length $N$ and $K=1.0$

\begin{tabular}{lll}
\hline$N$ & $C$ (standard) & $C$ (our method) \\
\hline 4 & 0.2242289 & 0.27152 \\
5 & 0.2319180 & 0.26768 \\
6 & 0.2371561 & 0.26640 \\
7 & 0.2408857 & 0.26567 \\
8 & 0.2436841 & 0.26524 \\
extrapolation: & & 0.262 \\
exact: & & 0.263272 \\
\hline
\end{tabular}

Table 2

Same as in table 1 , but for $K^{-1}=0.15$

\begin{tabular}{rll}
\hline $\mathrm{N}$ & $\mathrm{C}$ (standard) & $\mathrm{C}$ (our method) \\
\hline 4 & 0.1346858 & 0.23578 \\
5 & 0.0230826 & 0.15596 \\
6 & 0.1425179 & 0.13664 \\
7 & 0.0469311 & 0.12842 \\
8 & 0.1248113 & 0.11685 \\
9 & 0.0653559 & 0.11396 \\
10 & & 0.108 \\
extrapolation: & & 0.091 \\
exact: & & 0.082181 \\
\hline
\end{tabular}

ment, and equally between the last and next last:

$H_{0}+H^{\prime}=-\frac{1}{2} \sum_{i=1}^{N-1} K_{i}\left(\sigma_{i}^{x} \sigma_{i+1}^{x}+\sigma_{i}^{y} \sigma_{i+1}^{y}\right)$,

where $K_{1}=K_{N-1}=K+D$ and $K_{i}=K$ for all other $i$. We have solved this model by direct diagonalization where we determined $D$ by imposing $\left\langle\sigma_{1}^{x} \sigma_{2}^{x}\right\rangle$ $=\left\langle\overline{\sigma_{i}^{x} \sigma_{i+1}^{x}}\right\rangle$. Here we denote by $\bar{F}_{i}$ the average of $F_{i}$ over all $i$. We have done this for several values of $K$ and $N$. As a typical example we give in table 1 the results obtained for the specific heat (through a numerical differentiation of the energy) for $K=1.0$, together with the results from a standard calculation without the correction terms. For higher $K$-values (lower temperatures), where quantum effects become more important, our approximation becomes less exact; for the same $K$, however, the standard calculations lead to large even-odd fluctuations, which cannot be extrapolated. An example is presented in table 2 for $K=6.667$.

These preliminary successes are very promising for future applications to more complicated systems. They also justify further efforts with higher order approximations, e.g., by using more parameters to be determined simultaneously from self-consistency conditions.

One of the authors (G.K.) is indebted to the A. von Humboldt Foundation for a fellowship.

\section{References}

[1] See, e.g., C. Domb, Adv. Phys. 9 (1960) 149; J.S. Smart, Effective Field Theories of Magnetism (Saunders, Philadelphia, 1966).

[2] H.A. Bethe, Proc. R. Soc. London A 150 (1935) 552.

[3] M. Suzuki, J. Phys. Soc. Jpn. 57 (1988) 683.

[4] L.S. Campana, A. Caramico D'Auria, U. Esposito and G. Kamieniarz, Phys. Rev. B 41 (1990) 6733. 